\title{
Construction of a Neuroanatomical Shape Complex Atlas from 3D MRI Brain Structures
}

\author{
Ting Chen ${ }^{\mathrm{a}}$, Anand Rangarajan ${ }^{\mathrm{a}, *}$, Stephan J. Eisenschenk ${ }^{\mathrm{b}}$, Baba C. \\ Vemuri $^{\mathrm{a}, * *}$ \\ ${ }^{a}$ Department of CISE, University of Florida, Gainesville, FL 32611-6120,USA \\ ${ }^{b}$ Department of Neurology, University of Florida, Gainesville, FL 32611,USA
}

\begin{abstract}
Brain atlas construction has attracted significant attention lately in the neuroimaging community due to its application to the characterization of neuroanatomical shape abnormalities associated with various neurodegenerative diseases or neuropsychiatric disorders. Existing shape atlas construction techniques usually focus on the analysis of a single anatomical structure in which the important inter-structural information is lost. This paper proposes a novel technique for constructing a neuroanatomical shape complex atlas based on an information geometry framework. A shape complex is a collection of neighboring shapes - for example, the thalamus, amygdala and the hippocampus circuit - which may exhibit changes in shape across multiple structures during the progression of a disease. In this paper, we represent the boundaries of the entire shape complex using the zero level set of a distance transform function $S(\mathbf{x})$. We then re-derive the relationship between the stationary state wave function $\psi(\mathbf{x})$ of the Schrödinger equation $-\hbar^{2} \nabla^{2} \psi+\psi=0$ and the eikonal equation $\|\nabla S\|=1$ satisfied by any distance function. This leads to a one-to-one map (up to scale) between $\psi(\mathbf{x})$ and $S(\mathbf{x})$ via an explicit relationship. We further exploit this relationship by mapping $\psi(\mathbf{x})$ to a unit hypersphere whose Riemannian structure is fully known, thus effectively turn $\psi(\mathbf{x})$ into the square-root of a probability density

\footnotetext{
${ }^{*}$ Corresponding author

*** Principal corresponding author

Email addresses: tichen@cise.ufl.edu (Ting Chen), anand@cise.ufl.edu (Anand
} Rangarajan), stephan.eisenschenk@neurology.ufl.edu (Stephan J. Eisenschenk), vemuri@cise.ufl.edu (Baba C. Vemuri)
\end{abstract}


function. This allows us to make comparisons - using elegant, closed-form analytic expressions - between shape complexes represented as square-root densities. A shape complex atlas is constructed by computing the Karcher mean $\bar{\psi}(\mathbf{x})$ in the space of square-root densities and then inversely mapping it back to the space of distance transforms in order to realize the atlas shape. We demonstrate the shape complex atlas computation technique via a set of experiments on a population of brain MRI scans including controls and epilepsy patients with either right anterior medial temporal or left anterior medial temporal lobectomies.

Keywords: brain MRI, shape complex atlas, epilepsy, lobectomy, distance transform, Schrödinger equation, Karcher mean, level set, square-root density

\section{Introduction}

Human brain MRI analysis is an important problem due to its application in the diagnosis and treatment of neurological diseases. In this context, the construction of neuroanatomical atlases of the human brain is of particular interest and its importance has been emphasized in a number of recent studies (Yeo et al. (2008); Aljabar et al. (2009); Sabuncu et al. (2009); Shattuck et al. (2008)). In brief, an atlas provides a reference for a population of shapes/images which is useful in numerous applications: (i) statistical analysis of volumetric changes in control and patient populations, (ii) atlasguided segmentation of structures of interest which is needed in further diagnostic procedures, and (iii) automated detection of disease regions based on shape variations between the atlas and individual subjects. Most existing shape atlases are based on isolated, single anatomical shapes (Liu et al. (2008); Fletcher et al. (2004); Wang et al. (2006)) which do not contain any inter-structural information. For example, the spatial relationships among different neighboring structures may change due to the effect of non-uniform volume shrinkage or expansion of neighborhood structures. Furthermore, many neurological disorders are diagnosed by the structural abnormalities (e.g. volume change) ascribed to several brain structures rather than a single structure. Alzheimer's disease is an example of such a neurological disordera morphological marker for which is the enlargement of ventricles and the shrinkage of the entorhinal cortex, amygdala and hippocampi (Brice (2009)). Mania, which is most often associated with bipolar disorder serves as another 
example. In Strakowski et al. (1999), all the brain structures associated with the neural pathways were examined and the authors claimed that patients with mania have a significant overall volume difference in the regions including the thalamus, hippocampi and the amygdala. In Seidman et al. (1999), the authors concluded that the structural abnormalities in the thalamus and the amygdala-hippocampus regions represent remarkable anatomical vulnerabilities in schizophrenia subjects. Therefore, a neuroanatomical shape complex atlas which captures anatomical connectivity as well as inter-structural relationships is of primary clinical importance.

\section{Previous Work}

In the context of atlas construction for multiple brain structures, most of the efforts in the past were focused on building the full brain image probabilistic atlases. For instance, in Joshi et al. (2004); Avants and Gee (2004); Shi et al. (2010); Xie et al. (2010), several image atlas construction methods for the entire brain were proposed based on the acquisition of $3 \mathrm{D}$ brain $\mathrm{MR}$ scans. The traditional techniques for image atlas construction usually focus on developing effective image deformation methods to register a population of brain images. Subsequently (or in tandem), the atlas image is estimated as an average over the registered image population. More recent works are based on developing specific techniques for mean computation. For example, in the multi-regional atlas (Shi et al. (2010)), the region specific mean is estimated whereas in Xie et al. (2010), the geodesic mean of a population of brain images is computed via an intrinsic averaging method. The brain image atlas has its advantage in general brain analysis. The variations of the entire brain due to aging can be studied (Sabuncu et al. (2009)) and the segmentation of brain structures (via registration of the atlas) achieved (Joshi et al. (2004)) with the aid of the whole brain image atlas. However, image registration (and hence the analysis based on it) may not be accurate for particular structures of interest due to the misalignment caused by the overall deformation of the convoluted cortex with its gyrencephalic details. Furthermore, it is a non trivial task to extend these techniques to shape atlas construction. Consequently, we will forgo further discussion of image based atlases in this paper and restrict our focus to shape based atlas construction. A shape atlas is of great importance when the analysis is focused on a certain structure or a neural pathway containing several related structures in the brain: examples are the diseases associated with hippocampi and amygdala. 
Feature point-sets (or landmarks when specific identities are ascribed to the features) are one of the most common shape representations in the literature. Unbiased atlas construction of hippocampi via groupwise point-set registration of mixture model probability density functions is described in Chen et al. (2010b); Wang et al. (2008); Chui et al. (2004). While explicit point to point correspondences are recovered in Chui et al. (2004), informationtheoretic methodologies are adopted in Chen et al. (2010b); Wang et al. (2008) resulting in implicit correspondence. In Cootes et al. (2008), a statistical shape model is directly constructed on diffeomorphic deformation fields. Other methods that represent shapes in 2D using parametric curves and in 3D using parametric surfaces have also received considerable attention in the literature (Klassen et al. (2004); Sebastian et al. (2003)). Since intrinsic statistical shape analysis in the space of curves/surfaces is in general a non trivial task, methods using this representation have traditionally resorted to computing means etc. of spline parameters. In Styner et al. (2003), a characteristic 3D shape model dubbed the M-rep was proposed, and based on this representation, a mathematical characterization of the space of M-reps was developed. An atlas was then constructed in this space via computation of the geodesic mean of a population of shapes represented by M-reps (Fletcher et al. (2004)). Recent work in Liu et al. (2008) describes an interesting model using continuous spherical shapes to analyze the anatomical shape differences in the hippocampus of a control group and blind subjects.

To summarize, in all the techniques discussed thus far, the shape atlas is developed only for an isolated anatomical structure and it is difficult to generalize these methods to multiple connected anatomical structures in a neighborhood. A shape complex analysis algorithm was proposed in Cates et al. (2008), where the shapes are represented by point sets and the correspondences across the shape complexes are optimized via minimizing an entropy based cost function. Although this model leads to straightforward statistical shape analyses, it has to resort to a gradient descent strategy for the optimization. In Gorczowski et al. (2007); Qiu and Miller (2008) multiobject shape analysis frameworks were presented where each shape of the "multi-object" had an independent representation, and hence extra information on the structural relationships between different shapes needed to be maintained. In Litvin and Karl (2005), a multi-object shape distribution was used as a prior for 2D image segmentation, wherein the distribution of a set of shapes is defined as the average of the distribution corresponding to the individual shapes in the group. This method does extract features from 
a shape complex but this shape information is lost after averaging.

Before we turn to the actual approach in this work, we briefly describe the role of the correspondence problem in atlas estimation. Groupwise nonrigid registration is used in previous work Chen et al. (2010b); Wang et al. (2008); Chui et al. (2004) for atlas computation. If explicit point-to-point correspondences can be recovered from groupwise non-rigid registration, then an atlas can be subsequently computed by averaging over corresponding point locations. In contrast, in this work we quotient out an appropriate transformation (rigid, similarity, affine) prior to atlas computation in the space of distance transforms. Consequently, our approach avoids the correspondence problem but the computed atlas now depends on the spatial mapping that is quotiented out. Since distance transforms represent shapes implicitly (rather than explicitly), our approach can be used even in situations where topological differences exist - a common situation in shapes extracted from brain MRIwhereas correspondence-based approaches are notoriously problematic when topological differences are present.

In this paper, we propose a novel technique for constructing the atlas of a neuroanatomical shape complex consisting of multiple neuroanatomical structures where the inter-structural relationships are captured implicitly without any loss of information of any of the constituent structures. In our framework, we first use the zero level set of the distance transform function to represent the boundaries of the entire shape complex and based on the mathematical relationship derived in Section 3, we then map the distance transform functions to the space of square-root densities where a geodesic mean (atlas) is computed. Finally, the actual shape complex atlas is realized via the inverse map back to the space of distance transforms.

The key contributions of this paper are as follows: (i) We derive a novel relationship between the stationary state wave function $\psi(\mathbf{x})$ of the Schrödinger equation and the eikonal equation $\|\nabla S\|=1$ for the Euclidean distance transform problem, which serves as a "bridge" that connects the distance transform representation of the shape to the space of square-root-densities. (ii) The inter-structural relationships are well captured in our distance transform representation of the shape complex, which is of great clinical importance for studying the shape variations across multiple structures in both ontogenesis and in various neurological diseases. (iii) We represent shape complexes using square-root densities. Since the manifold of square-root density functions is a unit Hilbertian sphere and its geometry is well understood, it allows us to use intrinsic geometry to compare shape complexes and carry out a statistical 


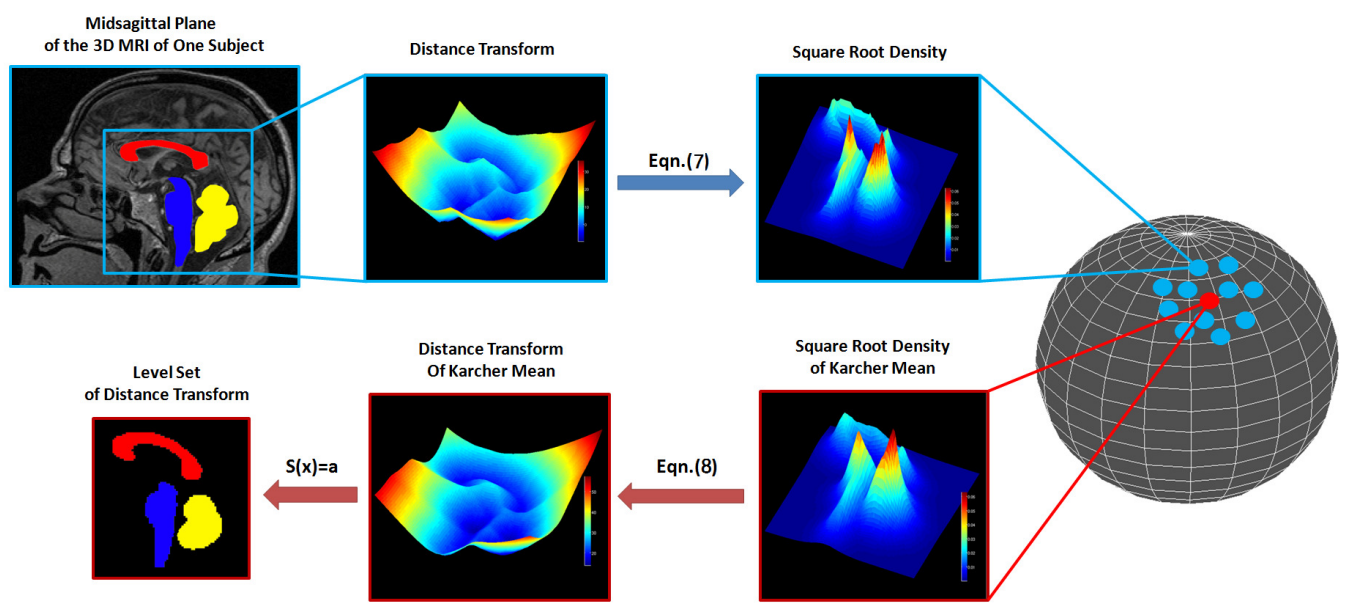

Figure 1: The flow chart of our framework. Here, we visualize the distance transform and square-root density in 2D case. Each sample data is represented by a blue point on the high dimensional sphere and the red point is the Karcher mean.

analysis of them.

The rest of the paper is organized as follows: In Section 3, we present the details of our shape complex atlas construction methodology. We demonstrate our technique in Section 4 on a 2D shape complex data set comprising the corpus callosum, brainstem and the cerebellum (taken from the midsagittal plane) and 3D brain structures including left/right hippocampus, entorhinal cortex, amygdala and thalamus. The data are from a population of $463 \mathrm{D}$ brain MR scans with all the neuroanatomical structures labeled by an expert neurologist.

\section{Shape Complex Atlas}

In this section, we derive the relationship between distance transform function and the square-root density representation, which allows us to model the shape complex in the square-root density space, perform the statistical analysis of the shapes and recover the mean shape back in the distance transform function space.

\subsection{From Distance Transforms to Square-Root Density Functions}

The distance transform is a well established technique for shape representation and has its advantage in capturing the details of complicated shapes. 
In our model, each shape complex data sample is represented by a distance transform function, the zero level set of which gives the individual boundaries of the various shapes constituting the shape complex. At least two decades of effort have gone into level set and distance function representations of shapes (Malladi et al. (1995); Sethian (1999); Osher and Fedkiw (2002); Sapiro (2001)) — the principal advantage being the ability to combine different shapes into a single scalar field representation. However, since variational and partial differential equation methods are at the foundation of level sets, it is a non-trivial task to employ statistical methods on scalar field distance function representations. Alternatively, there exists a class of methods that perform shape analysis by representing single shapes using probability density functions (Chen et al. (2010b); Litvin and Karl (2005); Squire and Caelli (2000)), and obtaining interesting and practical results. For instance, despite sacrificing the ability to represent a set of shapes or a shape complex, in this framework, the mean, variance and principal modes of the shape population are all easily computed. One of the main contributions of this paper is to successfully bridge the two disparate domains - variational and level set methods on the one hand and probabilistic methods on the other and directly obtain the density function of a shape complex from a distance transform function representation.

More recently, Pohl et al. (2007) embed signed distance functions into the linear space of LogOdds, where addition and scalar multiplication are in closed form. This manipulation allows the averaging and other operations of SDFs, however a monotonic function is needed to impose the SDF into the probability space of $[0,1]$. In this paper, we present a technique that is different from the aforementioned framework and provides an explicit function that maps the SDFs to the space of square-root densities. In Gurumoorthy and Rangarajan (2009), the authors begin by expressing the Euclidean distance function problem in a Schrödinger wave equation representation. They solve the Schrödinger wave equation instead of the corresponding static HamiltonJacobi equation to obtain the distance transform. While they emphasize that the main advantage of their approach is the linearity of the Schrödinger equation (as opposed to the non-linearity of the Hamilton-Jacobi equation), we wish to draw upon the obvious, historical precedent in quantum mechanics of motivating the Schrödinger wave function as a square-root density (Born (1926)). Inspired by this voluminous previous work, we adopt the interpretation of the stationary state Schrödinger wave function for the Euclidean distance transform as a square-root density. 
Generally speaking, our shape complex atlas construction technique constitutes the stages depicted in Fig. 1. We first represent the shape complex using a distance transform $S(\mathbf{x})$. Then, we derive a one-to-one map (up to scale) between $S(\mathbf{x})$ and the square-root representation $\psi(\mathbf{x})$ as in Eqn. (7) and convert the shape into the square-root density space, where statistical analysis can be easily performed. Finally, the atlas is computed by mapping the mean square-root density $\bar{\psi}(\mathbf{x})$ back into the distance transform space $\bar{S}(\mathbf{x})$ via Eqn. (8) followed by the extraction of the zero level set. In this procedure, the relationships in Eqn. (7) and Eqn. (8) play a key role.

We provide the derivation of the relationship between $S(\mathbf{x})$ and $\psi(\mathbf{x})$ below. Let $\psi(\mathbf{x})$ be the stationary state wave function (which is interpreted as square-root density) and let $\hbar$-Planck's constant-be a free parameter in this model. The static wave equation for the Euclidean distance function problem is

$$
\hbar^{2} \nabla^{2} \psi(\mathbf{x})=\psi(\mathbf{x}) .
$$

Please see Gurumoorthy and Rangarajan (2009) for a more detailed derivation.

Proposition 1. When $\psi(\boldsymbol{x})=\alpha \exp \left(\frac{-S(\boldsymbol{x})}{\hbar}\right)$ and satisfies Eqn. (1), $S(\boldsymbol{x})$ asymptotically satisfies the eikonal equation $\|\nabla S\|=1$ as $\hbar \rightarrow 0$. Here $\alpha$ is a normalization constant such that $\psi(\boldsymbol{x})$ is a square-root density, $\int_{\Omega}|\psi(\mathbf{x})|^{2} d \mathbf{x}=$ 1 where $\Omega$ is a bounded domain in $\mathbb{R}^{2}$ or $\mathbb{R}^{3}$.

Proof. From the definition of a square-root density, $\alpha^{2}=\frac{1}{\int_{\Omega} \exp \left(\frac{-2 S(\mathbf{x})}{\hbar}\right) d \mathbf{x}}$, which is a constant for each $S(\mathbf{x})$. Taking the $2 \mathrm{D}$ case as an example, when $\psi\left(x_{1}, x_{2}\right)=\alpha \exp \left(\frac{-S\left(x_{1}, x_{2}\right)}{\hbar}\right)$, we have for the first partials of $\psi\left(x_{1}, x_{2}\right)$ :

$$
\begin{aligned}
& \frac{\partial \psi}{\partial x_{1}}=-\frac{a}{\hbar} \exp \left(\frac{-S}{\hbar}\right) \frac{\partial S}{\partial x_{1}} \\
& \frac{\partial \psi}{\partial x_{2}}=-\frac{a}{\hbar} \exp \left(\frac{-S}{\hbar}\right) \frac{\partial S}{\partial x_{2}}
\end{aligned}
$$

and the second partials [required for the Laplacian in Eqn. (1)]:

$$
\begin{aligned}
& \frac{\partial^{2} \psi}{\partial x_{1}^{2}}=\frac{\alpha}{\hbar^{2}} \exp \left(\frac{-S}{\hbar}\right)\left(\frac{\partial S}{\partial x_{1}}\right)^{2}-\frac{\alpha}{\hbar} \exp \left(\frac{-S}{\hbar}\right) \frac{\partial^{2} S}{\partial x_{1}^{2}} \\
& \frac{\partial^{2} \psi}{\partial x_{2}^{2}}=\frac{\alpha}{\hbar^{2}} \exp \left(\frac{-S}{\hbar}\right)\left(\frac{\partial S}{\partial x_{2}}\right)^{2}-\frac{\alpha}{\hbar} \exp \left(\frac{-S}{\hbar}\right) \frac{\partial^{2} S}{\partial x_{2}^{2}}
\end{aligned}
$$


From Eqn. (1), we have $\left(\frac{\partial S}{\partial x_{1}}\right)^{2}+\left(\frac{\partial S}{\partial x_{2}}\right)^{2}-\hbar\left(\frac{\partial^{2} S}{\partial x_{1}^{2}}+\frac{\partial^{2} S}{\partial x_{2}^{2}}\right)=1$ which implies

$$
\|\nabla S\|^{2}-\hbar \nabla^{2} S=1
$$

Since $\nabla^{2} S$ is bounded, we obtain $\|\nabla S\|=1$ as $\hbar \rightarrow 0$.

The proposition above allows us to recover the distance transform function from the square-root density representation by computing the inverse map of

$$
\psi(\mathbf{x})=\alpha \exp \left(\frac{-S(\mathbf{x})}{\hbar}\right)
$$

which is

$$
S(\mathbf{x})=\hbar \log (\alpha)-\hbar \log (\psi(\mathbf{x})) .
$$

Note that Eqn. (7) is a surjective function, where at least two distance transforms are mapped to the same square-root density function. However, these distance transform functions only differ by a shift. Simple algebra will lead to the following: $S^{\prime}(\mathbf{x})=S(\mathbf{x})+c_{0}$, where $c_{0}$ is a constant and $S^{\prime}, S$ are mapped to the same $\psi$. This shifting effect is removed when we affine register all the shape complexes in the initialization process.

This important relationship builds a direct connection between the two realms, i.e. the level set framework and probability density functions. Hence, a shape complex of any complex topology can be represented using a single distance transform function and further statistical analysis of the shape population can be accomplished in the space of square-root densities - namely, the unit hypersphere - as a result of the transformation from the distance function to the square-root density representation.

\subsection{The Space of Square-Root Densities}

The square-root density has been widely used in the areas of computer vision and medical image analysis, see for example Peter et al. (2008); Goh et al. (2009); Srivastava et al. (2007). This is due to the fact that the resulting manifold is a unit sphere in Hilbert space, wherein both the inner products of the tangent vectors and the elements in the space are well defined. Hence, a variety of Riemannian operations are in closed-form since the space is a convex subset of a sphere in $L^{2}$.

Note that Eqn. (1) does not yield a solution that is a square-root density. However, it does build the relationship between exponentiated distance functions and the Schrödinger equation. Since the Schrödinger wave function 
has the property of being a square-root density (Born (1926)), we further restrict the solution to be in the square-root density space. The reasons for us to focus on the square-root density space rather than the exponentiated function space are as follows. Probability density functions are very useful shape representations as shown by several researchers in the literature. For instance, one can compute moments of the density and get global/local shape descriptors (Ho et al. (2009)) which cannot be achieved with an unnormalized exponentiated distance function. One can also match either the densities or their moments for the purpose of registration. Also, probability density functions allow us to relate our unknown smoothing parameter (Planck's constant) $\hbar$ to uncertainty. Furthermore, computing averages of un-normalized exponentiated functions is theoretically a difficult problem. The space of exponentiated functions is positive semidefinite whereas the square-root density space is the hypersphere which leads to a closed-form metric (and geodesic) that is efficient to compute.

For convenience, we reproduce the following well-known operations in the space of the high dimensional sphere. Let $\psi_{i}, i=1, \ldots, n$ be a set of squareroot densities in the space of such functions. Define $v \in T_{\psi_{1}} \psi$ as a vector in the tangent space of $\psi_{1}$.

- Geodesic Distance: $d\left(\psi_{1}, \psi_{2}\right)=\cos ^{-1}\left\langle\psi_{1}, \psi_{2}\right\rangle$

- Exponential Map: $\psi_{2}=\exp _{\psi_{1}}(v)=\cos (|v|) \psi_{1}+\sin (|v|) \frac{v}{|v|}$

- Log Map: $v=\log _{\psi_{1}}\left(\psi_{2}\right)=u \cos ^{-1}\left\langle\psi_{1}, \psi_{2}\right\rangle / \sqrt{\langle u, u\rangle}$, where $u=\psi_{2}-$ $\left\langle\psi_{2}, \psi_{1}\right\rangle \psi_{1}$

- Karcher Mean: $\bar{\psi}=\arg \min _{\psi \in \Psi} \sum_{i=1}^{n} d^{2}\left(\psi, \psi_{i}\right)$

Equipped with these basic tools, we are now able to construct an atlas for the shape complex by computing the Karcher mean of the given shape complex population in the space of the Hilbert sphere. We illustrate the idea of our framework on a simple example in Fig. 1. Note that the notion of atlas here corresponds to the mean computed using the $L^{2}$ norm. However, any norm is applicable in our framework. For example, we can envisage estimating the median of the population via the $L^{1}$ norm. Finally, with the square-root density representation, we can perform complete statistical analysis when required. 


\subsection{From Square-Root Density Functions to Distance Transforms}

In Section 3.1, we discussed the technique for mapping the shape complex represented by distance transform to the space of square-root density functions. The geodesic mean $\bar{\psi}(\mathbf{x})$ on the sphere of square-root densities is therefore computed from a population of shape complexes. Since each shape complex is finally represented by $\psi_{i}(\mathbf{x})=\alpha_{i} \exp \left(\frac{-S_{i}(\mathbf{x})}{\hbar}\right), i=1, \ldots, n$, it is valid to assume that their mean shape $\bar{\psi}(\mathbf{x})$ has the same representation as

each shape complex data sample, i.e. $\bar{\psi}(\mathbf{x})=\bar{\alpha} \exp \left(\frac{-\bar{S}(\mathbf{x})}{\hbar}\right)$. Therefore, the distance transform function of the mean shape (atlas) is given by

$$
\bar{S}(\mathbf{x})=\hbar \log (\bar{\alpha})-\hbar \log (\bar{\psi}(\mathbf{x}))
$$

Since Planck's constant $\hbar$ - a smoothing parameter in our framework - is set to a fixed value during the whole procedure, $\bar{\alpha}$ is the only parameter we need to estimate in order to recover $\bar{S}(\mathbf{x})$.

For each shape complex sample represented by $\psi_{i}, \alpha_{i}$ is the normalization parameter that imposes the square-root density property on $\psi_{i}$. Hence $\alpha_{i}$ is always greater than 0, i.e. $\alpha_{i} \in \mathbb{R}^{+}$. We denote the high dimensional Hilbert sphere by $\Phi$. Now, let $P=\mathbb{R}^{+} \times \Phi$ be the product manifold, and Eqn. (9) map the elements in $P$ to the space of distance transform functions. We evaluate $\bar{\psi}$ by computing the Karcher mean of $\left\{\psi_{i}\right\}$ on the unit Hilbert sphere and estimate $\bar{\alpha}$ via the Karcher mean of $\left\{\alpha_{i}\right\}$ in $\mathbb{R}^{+}$. The geodesic mean of $\left\{\psi_{i}\right\}$ has been investigated in the previous section and here we discuss how to compute $\bar{\alpha}$ in $\mathbb{R}^{+}$. It is known that the corresponding Riemannian distance between two elements $x, y \in \mathbb{R}^{+}$is $|\log x-\log y|$ (Moakher (2005); Barmpoutis and Vemuri (2009)). Therefore, the geodesic mean of $\left\{\alpha_{i}\right\}$ is simply the exponential of the average of $\log \alpha_{1}, \ldots, \log \alpha_{n}$, i.e.

$$
\bar{\alpha}=\exp \left(\frac{1}{n} \sum_{i=1}^{n} \log \alpha_{i}\right) .
$$

\section{Experiments}

In this section, we present several experiments demonstrating the performance of our algorithm on a population of real (shape complex) data. The images are a priori affine registered using an ITK-based mutual information registration algorithm (Thevenaz and Unser (2000)). Note that for visualization purposes, we transfer the labels of each structure in the shape complex 
by mapping the label image to our atlas. The transformation parameters of the mapping are computed by a non-rigid warping from a binary image of the shape complex template to the binary image estimated from the shape complex atlas.

\subsection{D Shape Complex Atlas}

To illustrate our technique, we begin with a $2 \mathrm{D}$ shape complex data set with the steps of our approach following the flowchart in Fig. 1. The 2D MR images are taken from the midsagittal plane of the 3D brain MRI with 3 structures (corpus callosum, brainstem and cerebellum) labeled by an expert neurologist. The flowchart contains the following major steps.

1. We estimate the distance transform function from each binary labeled image of the shape complex segmented by the expert neurologist (Dr. Eisenschenk). The zero level set of the distance transform captures the boundary contours of the shape complex.

2. From the distance transform representation of the shape complex, we compute its square-root density representation via Eqn. (7).

3. Each square-root density representation of the shape complex corresponds to a single point on the high dimensional sphere. In this Hilbertian sphere, the Karcher mean is directly computed. As a by product, statistical analysis such as the principal geodesic analysis (PGA) (Fletcher et al. (2004)), is performed.

4. The distance transform representation of the atlas is recovered from the geodesic mean (which is a square-root density function on the sphere) via Eqn. (8).

5. Finally, the shape contours are recovered from the $a$-level set of the distance transform function, where $a$ is estimated from Eqn. (9) and Eqn. (10). The need for this step is explained below.

In Fig. 2, we demonstrate the 2D shape complex atlas constructed from our algorithm with 8 given samples. The first row shows the distance transforms (step 4) of the atlas computed at different values of $\hbar$. The results of step 5 are illustrated in the second row. We can see that as $\hbar$ is increased, the smoothness of the recovered atlas shape contours increases.

Note that the signed distance transform was used in this experiment. Our algorithm is valid for both signed and unsigned distance transform functions. As shown in Fig. 3, whereas the square-root density of the unsigned distance 


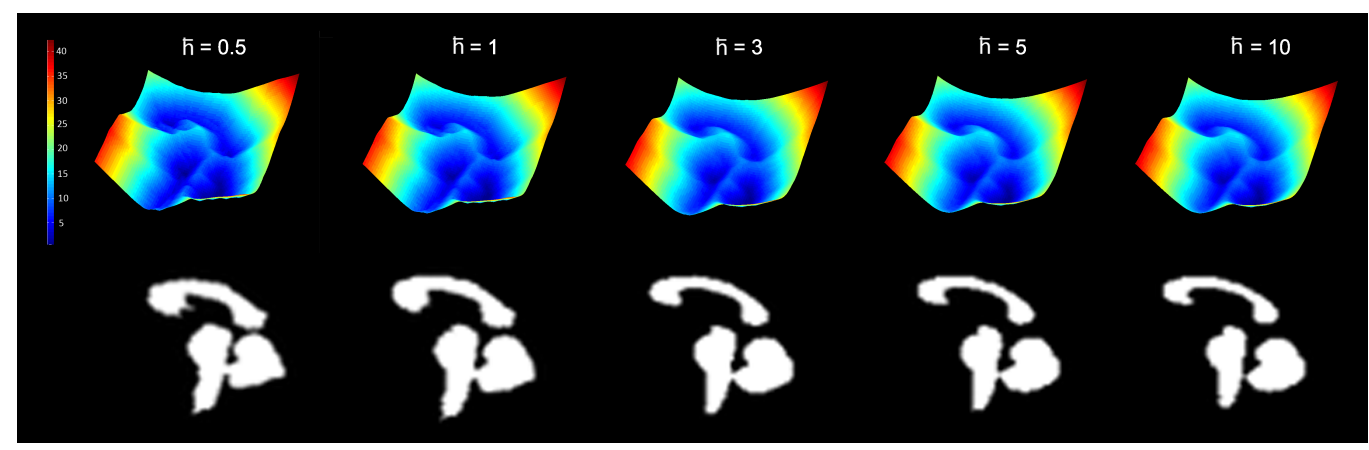

Figure 2: This figure depicts the Euclidean distance transforms and the exact shape contours of the 2D shape complex (corpus callosum, brainstem and cerebellum) atlas corresponding to different $\hbar$ values. As $\hbar$ increases, the atlas becomes more smooth.

transform has peaks on the shape boundary, its signed distance counterpart captures the skeleton of the shape complex. However, the signed distance transform representation is more robust in practice since we take the $a$-level set of the distance transform to recover the atlas shape contours. Due to numerical issues, we can not guarantee that the distance transform function generated from the inverse mapping has all the local minima on the same level set. Hence, the boundaries we extract from the $a$-level set might be noisy. We have not observed this to be a problem in the case of the signed distance transform.

\subsection{D Shape Complex Atlas}

Next, we apply our framework to a 3D shape complex data set (Fig. 4). This data set contains the 3D brain MRI from 32 controls and 14 patients with epilepsy ( 7 right and 7 left anterior medial temporal lobe epilepsy cases respectively). Epilepsy refers to a group of related neurological disorders characterized by recurrent seizures. In temporal lobe epilepsy, the hippocampus, amygdala, and parahippocampal regions are considered to be the epileptogenic focused structures. In this data set, we have the following 8 related structures labeled by the expert: left/right hippocampus, entorhinal cortex, amygdala and thalamus. The first experiment is to construct the shape complex atlas using our technique. We show the atlas constructed from the controls as the mean shape in three different angles of view in Fig. 5. The free parameter $\hbar$ acts as a smoothing/regularization term for atlas construction and is expected to act as an uncertainty control - similar to the role played by Planck's constant in quantum mechanics. We demonstrate 


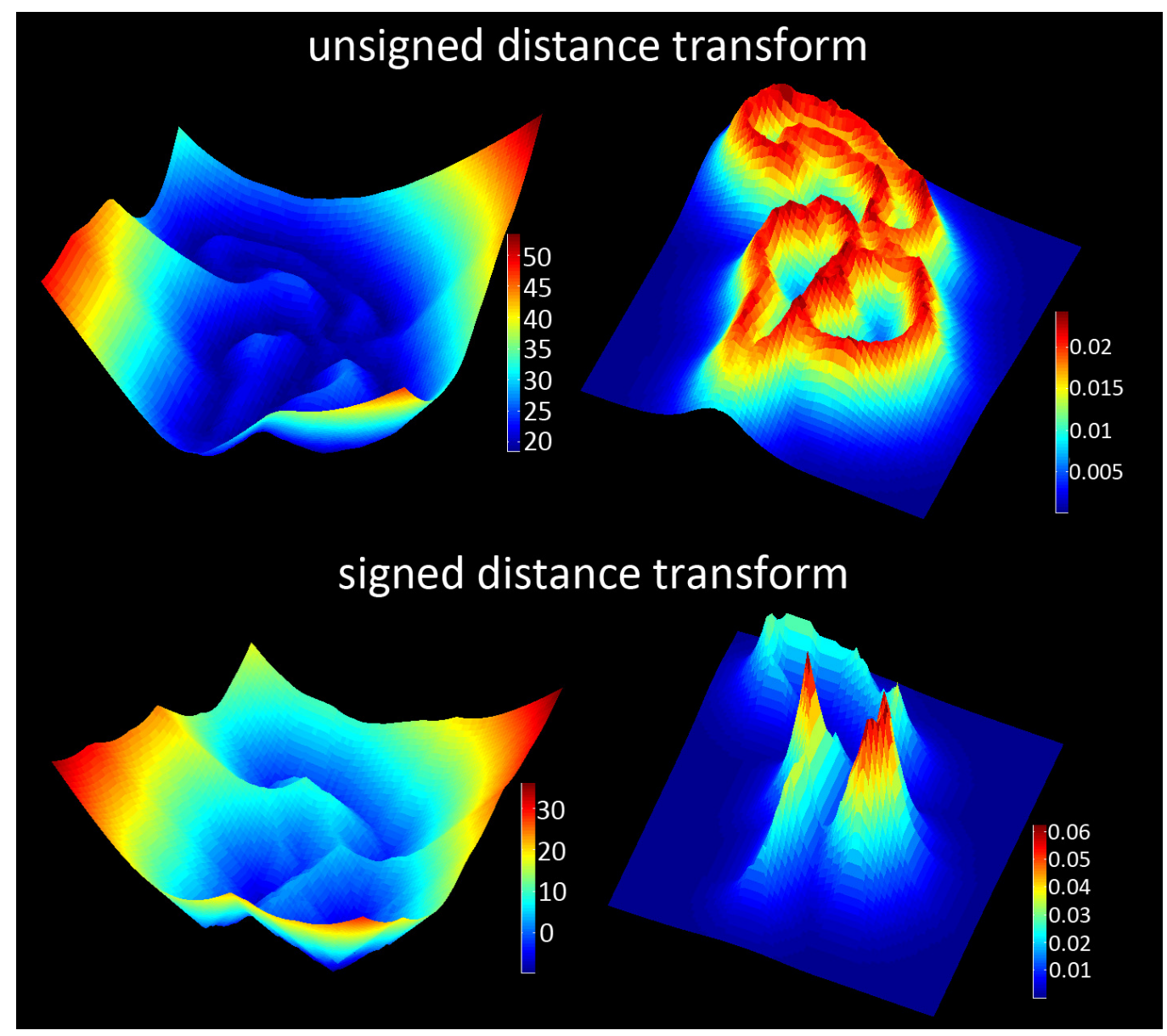

Figure 3: The first row of the figure shows the unsigned distance transform of the mean shape complex (atlas) on the left and its square-root density representation on the right. The second row shows the signed distance transform and its corresponding square-root density for the same data. 


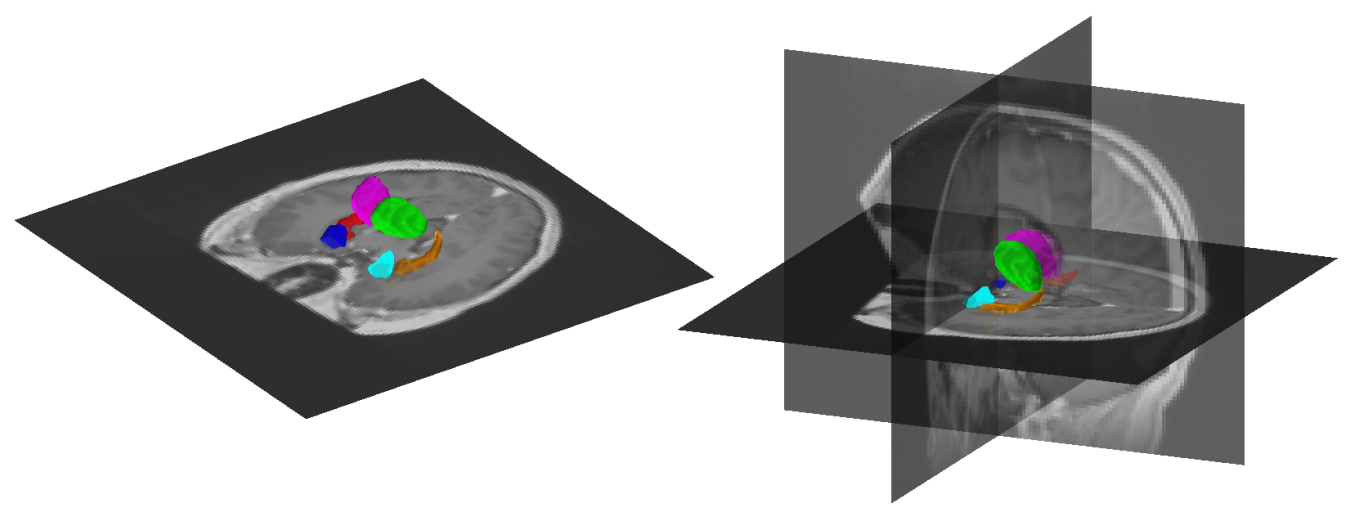

Figure 4: Two views of the 3D shape complex comprising 8 brain structures: the left/right hippocampus, entorhinal cortex, amygdala and thalamus.

the variation of the atlas when different $\hbar$ values are used. As $\hbar$ increases, the atlas becomes more smooth.

Since there is no analytical solution for computing the Karcher mean, we use a gradient-based approach (Srivastava et al. (2007)) to iteratively compute the geodesic mean of the square-root density functions. To demonstrate the convergence of Karcher mean computation, we estimate the error at each iteration as the $L_{2}$ norm of the difference between the current mean value and the one evaluated at the previous iteration. We show the errors w.r.t. the iteration number for different $\hbar$ values in Fig. 6 . The algorithm converges within 50 integrations for each setting, which is very efficient.

As the shape complex is represented using a square-root density, we are capable of performing a set of statistical analyses of the shapes. Applying principal geodesic analysis (PGA) (Fletcher et al. (2004)) to our data set, we recover the modes of deformation and the shape variation along the first and second principal directions as shown in Fig. 7.

\subsection{Shape Variation Analysis}

In statistical analysis of shape variation, such as the study of structural deformations occurring in the epilepsy process, volume based analysis has been popular for decades (Gerig et al. (2001); Csernansky et al. (1998)). It has been shown that brain structure volume shrinkage occurs in epilepsy patients (Marsh et al. (1997)). Therefore, to investigate epileptic brain structure shape variations, we designed the following experiments. We randomly take 7 of the control subjects and use them for testing. The remaining 25 


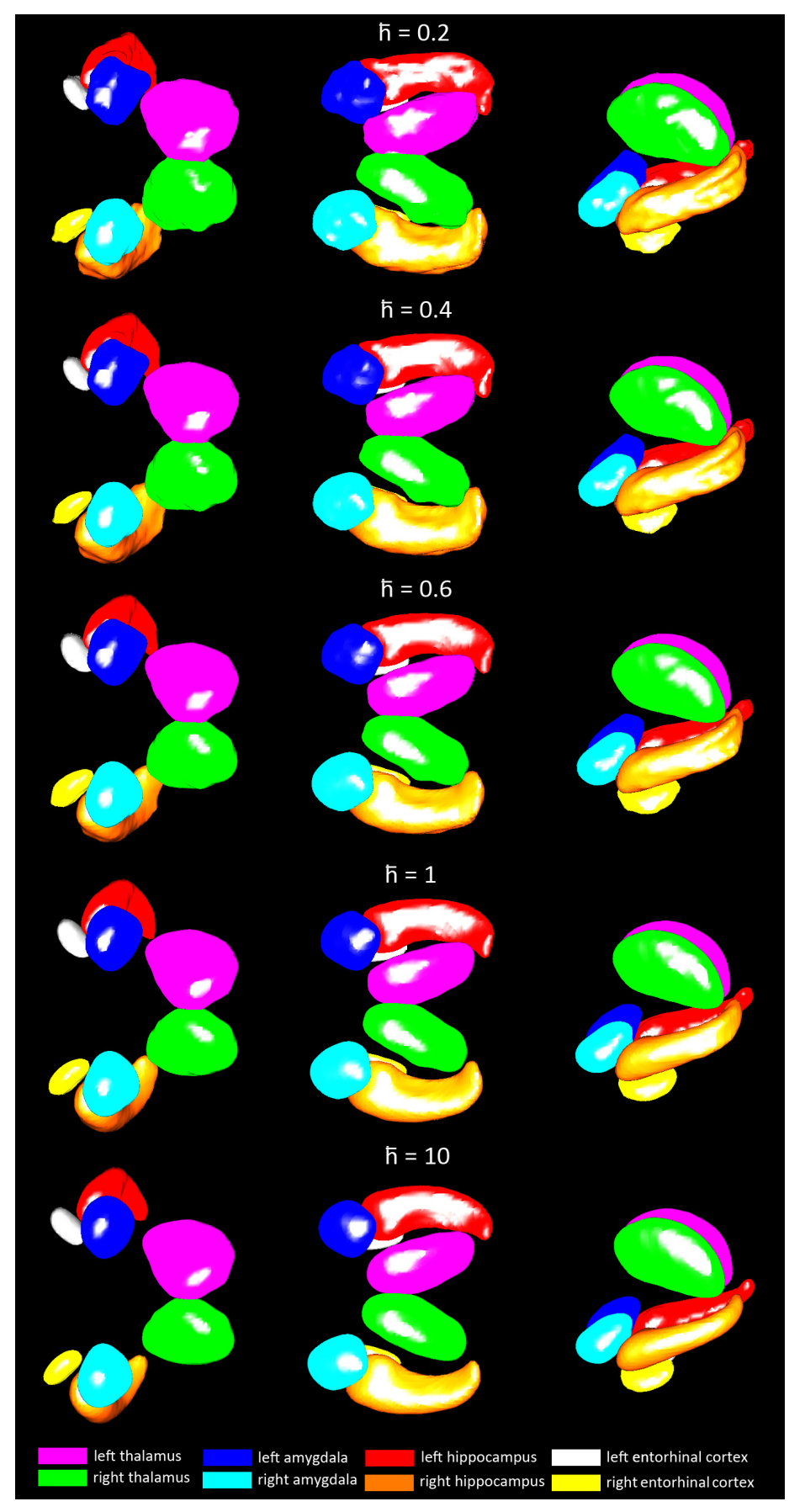

Figure 5: This figure shows the three views of the 3D shape complex atlas corresponding to different $\hbar$ values in each row. As $\hbar$ increases, the atlas becomes more smooth. 


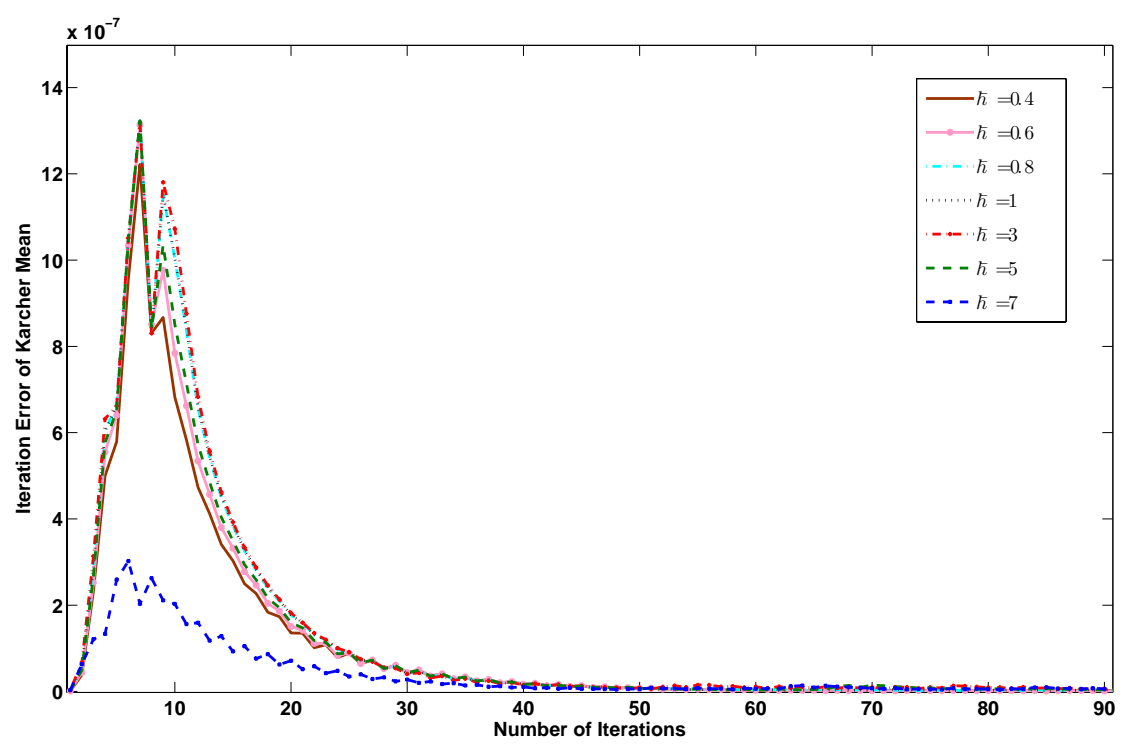

Figure 6: Error of the Karcher mean iteration for different $\hbar$ values.

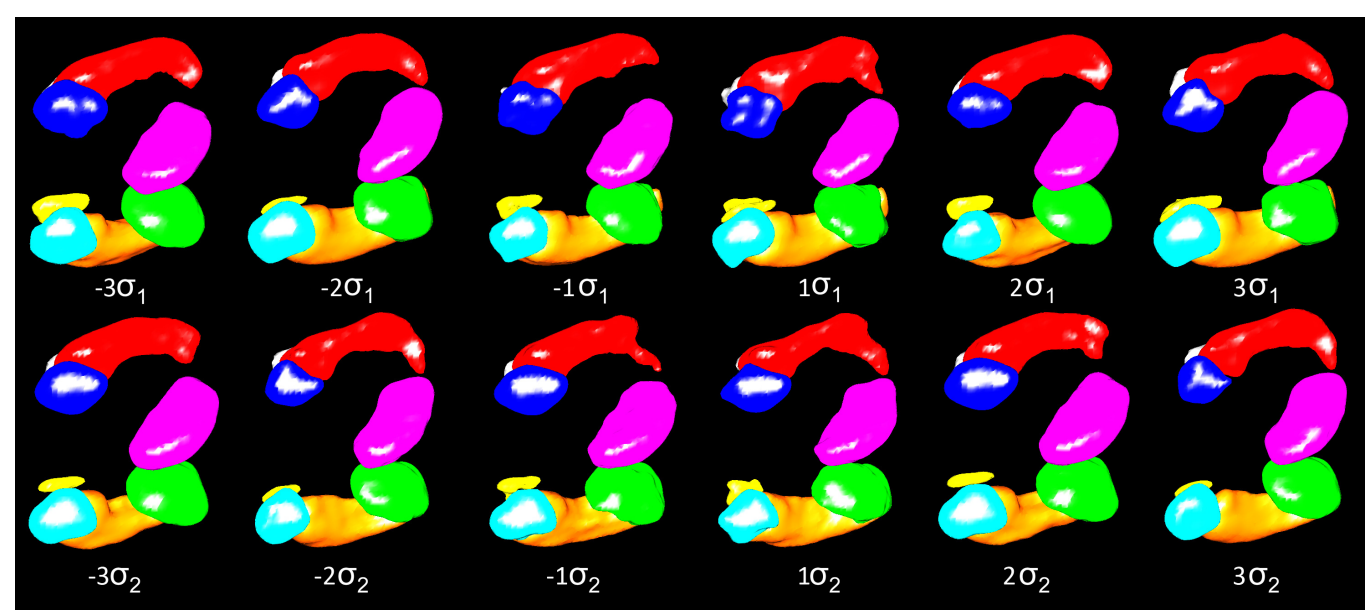

Figure 7: The shape variations along the first and second principal directions. Here $\hbar=0.6$. 
samples (data) are used to construct the atlas. The test data then contains 7 control, 7 LATL and 7 RATL samples. We compute the shape variations between the testing data and the atlas as follows:

$$
\begin{gathered}
\text { Volume Index }=\frac{\text { Volume }(\text { subject })}{\text { Volume }(\text { atlas })}, \\
\text { Similarity Index }=2 \frac{\text { Volume }(\text { subject } \cap \text { atlas })}{\text { Volume }(\text { subject })+\text { Volume }(\text { atlas })}, \text { and } \\
\text { Difference Index }=\frac{2 \mid \text { Volume }(\text { subject })-\text { Volume }(\text { atlas }) \mid}{\text { Volume }(\text { subject })+\text { Volume }(\text { atlas })},
\end{gathered}
$$

where the Volume Index (also known as the Dice Coefficient) denotes the entire volume change between the test subject w.r.t. the atlas, the Similarity Index indicates the region of overlap between the subject and the atlas, and the Difference Index shows the difference of the two in terms of volume size. The results are listed in Tables 1,2 and 3. Note that large brain volume shrinkage is observed for the epilepsy patients compared to the controls. To better illustrate these results, we plot the overlaps of the testing shape complexes (in gray) and the atlas (in colored mesh) in Fig. 9. Studies have shown that distinguishing between LATL and RATL in epilepsy is a hard problem and that we need sophisticated features in order to automatically classify them (Kodipaka et al. (2007)). Tables 2 and 3 indicate that the indices for both LATL and RATL are similar hence the left and right anterior medial temporal lobe focuses are indistinguishable w.r.t. volume based analysis. A promising immediate avenue for future research (following Kodipaka et al. (2007)) is to utilize the histogram of the deformation field between the shape complex of the subject and the atlas for further analysis.

In our approach, a single distance transform is used to represent a shape complex. Consequently, despite going against the grain of our philosophy, we can build atlases for the individual brain structures in the shape complex. To demonstrate this "by-product" of our algorithm and the effect of epilepsy on each brain structure, we build the atlas of each structure separately and compute the Volume Index between the atlas and the test data for each structure. The results are shown in Fig. 8. For LATL, we observe larger volume shrinkage for the structures in left brain than in the right brain while for RATL, right brain structures experience larger atrophy. This is obviously anecdotal but indicates the need for more detailed empirical analyses on larger data set. 


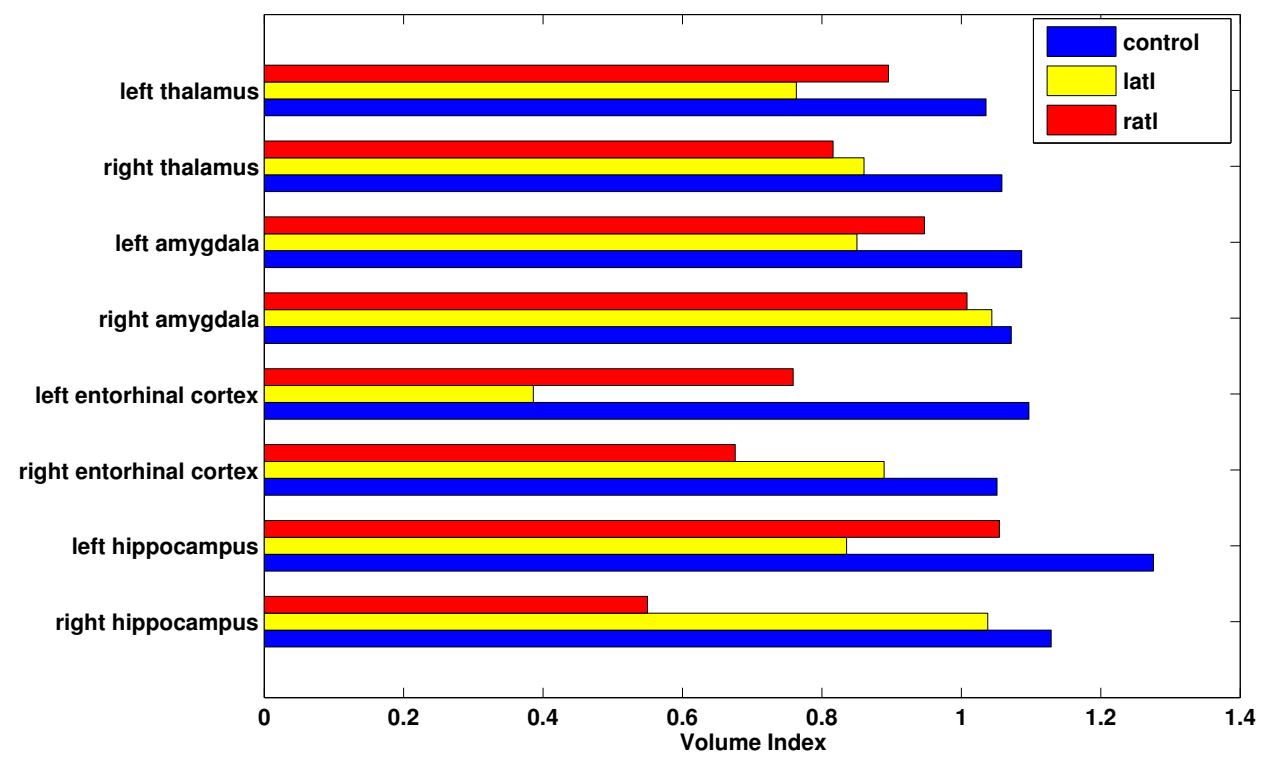

Figure 8: The average Volume Index for each structure and for the test data set.

Our atlas construction algorithm was implemented in Matlab ${ }^{\circledR}$ on a $2.80 \mathrm{GHZ}$ Intel Core(TM) i7 CPU PC. It takes less than 2 seconds to construct an atlas from 25 labeled brain MRI with the dimensions of the ROI being $71 \times 65 \times 79$. This serves to "loosely" illustrate the computational time involved.

\section{Conclusions}

The raison d'être for our new approach is the premise that atlases of nearby 3D MRI brain structures should use an integrated representation in which individual structures are not compromised. To this end, we designed a neuroanatomical atlas construction framework (and algorithm) for shape complex data. We derived and utilized the relationships between the 


\begin{tabular}{|c|c|c|c|}
\hline Shape Complex & \multicolumn{3}{|c|}{ Control } \\
\hline \hline subject ID & Volume Index & Similarity Index & Difference Index \\
\hline 1 & 1.0882 & 0.8055 & 0.0845 \\
\hline 2 & 1.0435 & 0.8348 & 0.0426 \\
\hline 3 & 0.9130 & 0.8385 & 0.0910 \\
\hline 4 & 0.9178 & 0.8349 & 0.0857 \\
\hline 5 & 0.9479 & 0.8186 & 0.0535 \\
\hline 6 & 0.9262 & 0.8087 & 0.0767 \\
\hline 7 & 0.8853 & 0.7955 & 0.1217 \\
\hline \hline mean & $\mathbf{0 . 9 6 0 3}$ & $\mathbf{0 . 8 1 9 5}$ & $\mathbf{0 . 0 7 9 4}$ \\
\hline std. & 0.0756 & 0.0169 & 0.0259 \\
\hline
\end{tabular}

Table 1: Shape variations for the control subjects compared to the atlas.

\begin{tabular}{|c|c|c|c|}
\hline Shape Complex & \multicolumn{3}{|c|}{ LATL } \\
\hline \hline subject ID & Volume Index & Similarity Index & Difference Index \\
\hline 1 & 0.9337 & 0.7478 & 0.0686 \\
\hline 2 & 0.8465 & 0.7280 & 0.1662 \\
\hline 3 & 0.8983 & 0.7403 & 0.1072 \\
\hline 4 & 0.8250 & 0.7254 & 0.1918 \\
\hline 5 & 0.8854 & 0.7975 & 0.1216 \\
\hline 6 & 0.5716 & 0.6465 & 0.5451 \\
\hline 7 & 0.8985 & 0.7824 & 0.1070 \\
\hline \hline mean & $\mathbf{0 . 8 3 7 0}$ & $\mathbf{0 . 7 3 8 3}$ & $\mathbf{0 . 1 8 6 8}$ \\
\hline std. & 0.1224 & 0.0487 & 0.1632 \\
\hline
\end{tabular}

Table 2: Shape variations for the LATL subjects compared to the atlas. 


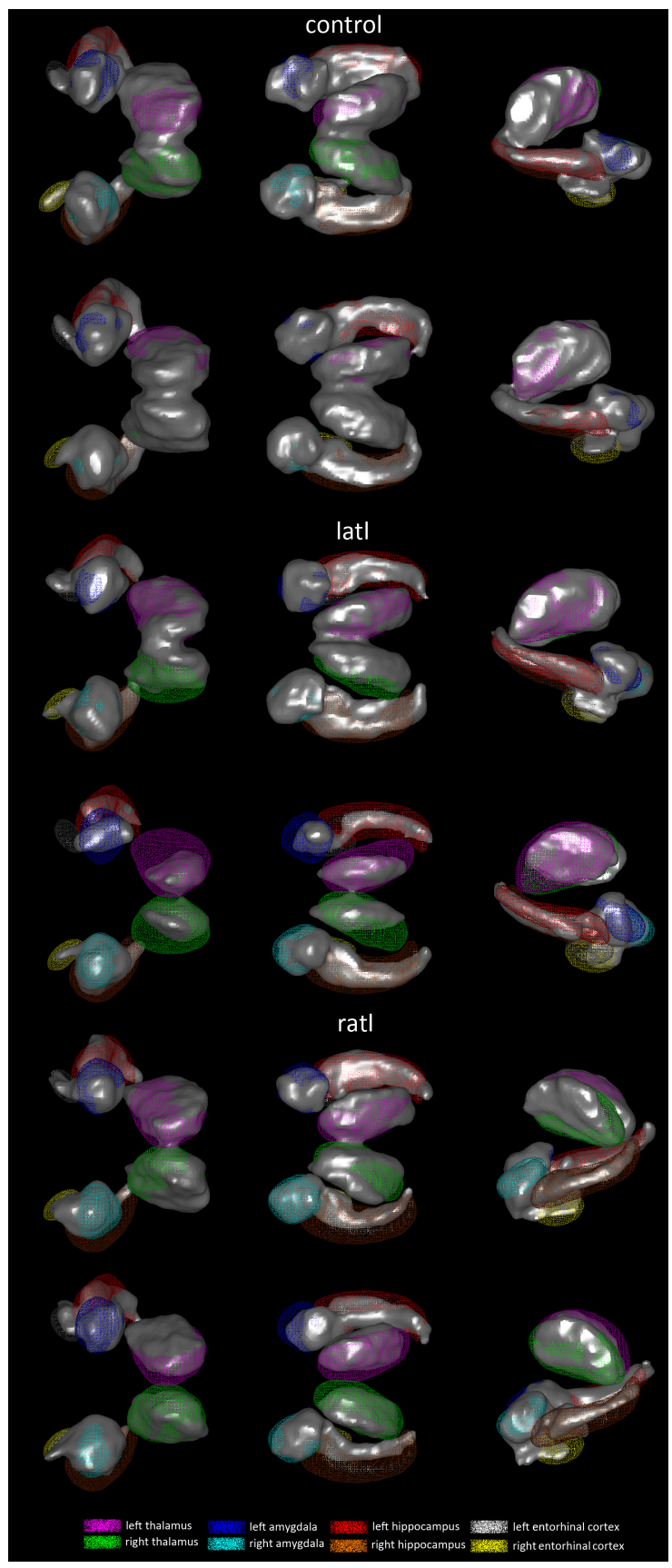

Figure 9: The overlap between the atlas and 2 examples from the control, LATL and RATL data set respectively. The atlas is depicted using a colored mesh and the test data is shown in gray. 


\begin{tabular}{|c|c|c|c|}
\hline Shape Complex & \multicolumn{3}{|c|}{ RATL } \\
\hline \hline subject ID & Volume Index & Similarity Index & Difference Index \\
\hline 1 & 0.8614 & 0.7819 & 0.1489 \\
\hline 2 & 0.7559 & 0.7154 & 0.2781 \\
\hline 3 & 0.8434 & 0.7501 & 0.1699 \\
\hline 4 & 0.8816 & 0.7899 & 0.1258 \\
\hline 5 & 0.9062 & 0.7680 & 0.0984 \\
\hline 6 & 0.8865 & 0.8057 & 0.1203 \\
\hline 7 & 0.7628 & 0.7582 & 0.2691 \\
\hline \hline mean & $\mathbf{0 . 8 4 2 6}$ & $\mathbf{0 . 7 6 9 6}$ & $\mathbf{0 . 1 7 2 9}$ \\
\hline std. & 0.0602 & 0.0305 & 0.0724 \\
\hline
\end{tabular}

Table 3: Shape variations for the RATL subjects compared to the atlas.

Euclidean distance transform and the square-root density Schrödinger wave function representation and this successfully builds on a connection between the realms of the level set framework and probability density functions. Our model is not only capable of preserving the spatial relationships among the different structures in the shape complex but also of carrying out a variety of statistical analyses of the shape complex population. We experimentally demonstrate the shape complex atlas computation algorithm on a population of brain MRI scans. We also present modes of variation from the computed atlas (from both the shape complex and individual structures) for the control population and for LATL and RATL patients.

Most existing atlas construction methods are based on registering shapes/images to a common space and estimating the mean. Topology preserving deformation techniques (Wang et al. (2003); Yanovsky et al. (2007)) can be used to register the data, however, the mean shape/image evaluation cannot guarantee the final topology. That is, the boundaries of adjacent structures will be blurred and merged during the averaging. Since the atlas construction process in our algorithm is also based on mean computation, the atlas topology is therefore not guaranteed. Recently, several techniques have been proposed to construct a topologically meaningful atlas from nearby data samples (Gerber et al. (2009); Xie et al. (2010); Hamm et al. (2010)). While these methods obtain good empirical results w.r.t. shape topology, there is no guarantee that topology is preserved (when multiple shapes are present). This is clearly a good avenue for future research. 


\section{Acknowledgments}

This work was partially supported by NSF RI-IIS 0954032 (PI: BCV) and NSF IIS 1143963 (PI: AR) An abbreviated version of this paper was first published in Chen et al. (2010a).

\section{References}

Aljabar, P., Heckemann, R.A., Hammers, A., Hajnal, J.V., Rueckert, D., 2009. Multi-atlas based segmentation of brain images: Atlas selection and its effect on accuracy. NeuroImage 46, 726-738.

Avants, B., Gee, J.C., 2004. Geodesic estimation for large deformation anatomical shape averaging and interpolation. Neuroimage 23, S139-150.

Barmpoutis, A., Vemuri, B.C., 2009. Groupwise registration and atlas construction of 4th-order tensor fields using the $\mathbb{R}^{+}$Riemannian metric. Medical Image Computing and Computer-Assisted Intervention (MICCAI) LNCS 5761, 640-647.

Born, M., 1926. Zur Quantenmechanik der Stoßvorgänge. Zeitschrift für Physik A: Hadrons and Nuclei 37, 863-867.

Brice, J., 2009. Volumetric MRI produces early warning on Alzheimer's disease. Diagnostic Imaging Europe .

Cates, J., Fletcher, P.T., Styner, M., Hazlett, H.C., Whitaker, R., 2008. Particle-based shape analysis of multi-object complexes. Medical Image Computing and Computer-Assisted Intervention (MICCAI) 11, 477-485.

Chen, T., Rangarajan, A., Eisenschenk, S.J., Vemuri, B.C., 2010a. Construction of neuroanatomical shape complex atlas from 3D brain MRI. Medical Image Computing and Computer-Assisted Intervention (MICCAI) 13(3), $65-72$.

Chen, T., Vemuri, B.C., Rangarajan, A., Eisenschenk, S.J., 2010b. Groupwise point-set registration using a novel CDF-based Havrda-Charvát divergence. International Journal of Computer Vision 86, 111-124.

Chui, H., Rangarajan, A., Zhang, J., Leonard, C.M., 2004. Unsupervised learning of an atlas from unlabeled point-sets. IEEE Transactions on Pattern Analysis and Machine Intelligence 26, 160-172. 
Cootes, T.F., Twining, C.J., Babalola, K.O., Taylor, C.J., 2008. Diffeomorphic statistical shape models. Image and Vision Computing 26, 326-332.

Csernansky, J.G., Joshi, S., Wang, L., Haller, J.W., Gado, M., Miller, J.P., Grenander, U., Miller, M.I., 1998. Hippocampal morphometry in schizophrenia by high dimensional brain mapping. Neurobiology 95(19), $11406-11411$.

Fletcher, P., Lu, C., Pizer, S., Joshi, S., 2004. Principal geodesic analysis for the study of nonlinear statistics of shape. IEEE Transactions on Medical Imaging 23, 995-1005.

Gerber, S., Tasdizen, T., Joshi, S., Whitaker, R., 2009. On the manifold structure of the space of brain images. Medical Image Computing and Computer-Assisted Intervention (MICCAI) 12, 305-312.

Gerig, G., Styner, M., Shenton, M.E., Lieberman, J.A., 2001. Shape vs. size: Improved understanding of the morphology of brain structures. Medical Image Computing and Computer-Assisted Intervention (MICCAI) 4, 2432 .

Goh, A., Lenglet, C., Thompson, P.M., Vidal, R., 2009. A nonparametric Riemannian framework for processing high angular resolution diffusion images (HARDI). IEEE Conference on Computer Vision and Pattern Recognition (CVPR) , 2496-2503.

Gorczowski, K., Styner, M., Jeong, J., Marron, J.S., Piven, J., Hazlett, H.C., Pizer, S.M., Gerig, G., 2007. Statistical shape analysis of multi-object complexes. IEEE Conference on Computer Vision and Pattern Recognition (CVPR) 17-22, 1-8.

Gurumoorthy, K., Rangarajan, A., 2009. A Schrödinger equation for the fast computation of approximate Euclidean distance functions. Scale Space and Variational Methods in Computer Vision (SSVM) 5567, 100-111.

Hamm, J., Ye, D.H., Verma, R., Davatzikos, C., 2010. GRAM: A framework for geodesic registration on anatomical manifolds. Medical Image Analysis $14(5), 633-642$. 
Ho, J., Peter, A., Rangarajan, A., Yang, M., 2009. An algebraic approach to affine registration of point sets. International Conference on Computer Vision (ICCV), 1335-1340.

Joshi, S., Davis, B., Jomier, M., Gerig, G., 2004. Unbiased diffeomorphic atlas construction for computational anatomy. Neuroimage 23, S151-160.

Klassen, E., Srivastava, A., Mio, W., Joshi, S.H., 2004. Analysis of planar shapes using geodesic paths on shape spaces. IEEE Transactions on Pattern Analysis and Machine Intelligence 26, 372-383.

Kodipaka, S., Vemuri, B.C., Rangarajan, A., Leonard, C.M., Schmallfuss, I., Eisenschenk, S., 2007. Kernel Fisher discriminant for shape-based classification in Epilepsy. Medical Image Analysis 11(1), 79-90.

Litvin, A., Karl, W.C., 2005. Coupled shape distribution-based segmentation of multiple objects. Information Processing in Medical Imaging , 345-356.

Liu, X., Mio, W., Shi, Y., Dinov, I., Liu, X., Leporé, N., Leporé, F., Fortin, M., Voss, P., Thompson, P.M., 2008. Models of normal variation and local contrasts in hippocampal anatomy. Medical Image Computing and Computer-Assisted Intervention (MICCAI) 11, 407-415.

Malladi, R., Sethian, J.A., Vemuri, B.C., 1995. Shape modeling with front propagation: a level set approach. IEEE Transactions on Pattern Analysis and Machine Intelligence 17, 158-175.

Marsh, L., Morrell, M.J., Shear, P.K., Sullivan, E.V., Freeman, H., Marie, A., Lim, K.O., Pfefferbaum, A., 1997. Cortical and hippocampal volume deficits in temporal lobe epilepsy. Epilepsia 38, 576-587.

Moakher, M., 2005. A differential geometric approach to the geometric mean of symmetric positive-definite matrices. SIAM Journal on Matrix Analysis and Applications 26, 735-747.

Osher, S., Fedkiw, R., 2002. Level Set Methods and Dynamic Implicit Surfaces. Springer, New York.

Peter, A., Rangarajan, A., Ho, J., 2008. Shape L'Âne Rouge: Sliding wavelets for indexing and retrieval. IEEE Conference on Computer Vision and Pattern Recognition (CVPR), 1-8. 
Pohl, K.M., Fisher, J., Shenton, M., McCarley, R.W., Grimson, W.E., Kikinis, R., Wells, W.M., 2007. Using the logarithm of odds to define a vector space on probabilistic atlases. Medical Image Analysis 11(5), 465-477.

Qiu, A., Miller, M.I., 2008. Multi-structure network shape analysis via normal surface momentum maps. NeuroImage 42, 1430-1438.

Sabuncu, M.R., Balci, S.K., Shenton, M.E., Golland, P., 2009. Image-driven population analysis through mixture modeling. IEEE Transactions on Medical Imaging 28, 1473-1487.

Sapiro, G., 2001. Geometric Partial Differential Equations and Image Analysis. Cambridge University Press.

Sebastian, T., Klein, P., Kimia, B., 2003. On aligning curves. IEEE Transactions on Pattern Analysis and Machine Intelligence 25, 116-125.

Seidman, L.J., Faraone, S.V., Goldstein, J.M., Goodman, J.M., Kremen, W.S., Toomey, R., Tourville, J., Kennedy, D., Makris, N., Caviness, V.S., Tsuang, M.T., 1999. Thalamic and amygdala hippocampal volume reductions in first-degree relatives of patients with schizophrenia: an MRI-based morphometric analysis. Biological Psychiatry 46, 941-954.

Sethian, J.A., 1999. Level Set Methods and Fast Marching Methods: Evolving Interfaces in Computational Geometry, Fluid Mechanics, Computer Vision, and Materials Science. Cambridge University Press.

Shattuck, D.W., Mirza, M., Adisetiyo, V., Hojatkashani, C., Salamon, G., Narr, K.L., Poldrack, R.A., Bilder, R.M., Toga, A.W., 2008. Construction of a 3D probabilistic atlas of human cortical structures. NeuroImage 39, 1064-1080.

Shi, F., Yap, P., Fan, Y., Gilmore, J.H., Lin, W., Shen, D., 2010. Construction of multi-region-multi-reference atlases for neonatal brain MRI segmentation. Neuroimage 51, 684-693.

Squire, D.M., Caelli, T.M., 2000. Invariance signature: Characterizing contours by their departures from invariance. Computer Vision and Image Understanding 77, 284-316. 
Srivastava, A., Jermyn, I., S.Joshi, 2007. Riemannian analysis of probability density functions with applications in vision. IEEE Conference on Computer Vision and Pattern Recognition (CVPR), 1-8.

Strakowski, S.M., DelBello, M.P., Sax, K.W., Zimmerman, M.E., Shear, P.K., Hawkins, J.M., Larson, E.R., 1999. Brain magnetic resonance imaging of structural abnormalities in bipolar disorder. Archives of General Psychiatry 56, 254-260.

Styner, M., Gerig, G., Lieberman, J., Jones, D., Weinberger, D., 2003. Statistical shape analysis of neuroanatomical structures based on medial models. Medical Image Analysis 7, 207-220.

Thevenaz, P., Unser, M., 2000. Optimization of mutual information for multiresolution image registration. IEEE Transactions on Image Processing 9, 2083-2099.

Wang, F., Vemuri, B.C., Rangarajan, A., Eisenschenk, S.J., 2008. Simultaneous nonrigid registration of multiple point sets and atlas construction. IEEE Transactions on Pattern Analysis and Machine Intelligence 30, 20112022.

Wang, L., Miller, J.P., Gado, M.H., McKeel, D.W., Rothermich, M., Miller, M.I., Morris, J.C., Csernansky, J.G., 2006. Abnormalities of hippocampal surface structure on very mild dementia of the Alzheimer type. NeuroImage $30,52-60$.

Wang, S., Ji, J.X., Liang, Z., 2003. Landmark-based shape deformation with topology-preserving constraints. IEEE International Conference on Computer Vision (ICCV) 2, 923-930.

Xie, Y., Ho, J., Vemuri, B.C., 2010. Image atlas construction via intrinsic averaging on the manifold of images. IEEE Conference on Computer Vision and Pattern Recognition (CVPR), 2933-2939.

Yanovsky, I., Thompson, P.M., Osher, S., Leow, A.D., 2007. Topology preserving log-unbiased nonlinear image registration: Theory and implementation. IEEE Conference on Computer Vision and Pattern Recognition (CVPR) , 1-8. 
Yeo, B.T.T., Sabuncu, M.R., Desikan, R., Fischl, B., Golland, P., 2008. Effects of registration regularization and atlas sharpness on segmentation accuracy. Medical Image Analysis 12, 603-615. 